\title{
Chaotic mixing of a competitive--consecutive reaction
}

\author{
Stephen M. Cox ${ }^{1}$ \\ School of Mathematical Sciences, University of Nottingham, University Park, \\ Nottingham NG7 2RD, United Kingdom
}

\begin{abstract}
The evolution of a competitive-consecutive chemical reaction is computed numerically in a two-dimensional chaotic fluid flow with initially segregated reactants. Results from numerical simulations are used to evaluate a variety of reduced models commonly adopted to model the full advection-reaction-diffusion problem. Particular emphasis is placed upon fast reactions, where the yield varies most significantly with Péclet number (the ratio of diffusive to advective time scales). When effects of the fluid mechanical mixing are strongest, we find that the yield of the reaction is underestimated by a one-dimensional lamellar model that ignores the effects of fluid mixing, but overestimated by two other lamellar models that include fluid mixing.
\end{abstract}

Key words: chaotic advection, competitive-consecutive chemical reaction, series-parallel chemical reaction, reaction-diffusion system

PACS: 47.15.-x, 47.52.+j, 47.47.-n, 82.40.-g

$\overline{1}$ e-mail: stephen.cox@nottingham.ac.uk; tel: +44 115951 4930; fax: +44 115 9514951 


\section{Introduction}

The evolution of chemical reactions between initially segregated reactants is strongly influenced by the scale of segregation $[1,2]$. When the reactions take place in a liquid phase that is stirred continuously, their progress correspondingly depends strongly upon the details of the fluid mechanical mixing [1-9]. In this paper, we examine the influence of fluid mixing upon a competitiveconsecutive (or series-parallel) reaction, $A+B \rightarrow R, B+R \rightarrow S$ [1], that takes place in a two-dimensional, laminar, chaotic fluid flow. (Although in applications mixing is often generated through turbulent flow, laminar flow is more appropriate for highly viscous fluids or for delicate polymers or suspensions, for instance [9].) We report numerical simulations of the simultaneous advection, reaction and diffusion of the various chemical species. Accurate simulations, even in two space dimensions, remain a significant computational challenge, because the chaotic flow generates structures whose spatial scales decrease exponentially with time, thereby rapidly reaching any fixed spatial resolution used in the numerics, or forcing adaptive grid refinements which correspondingly involve prohibitive computational expense. In the presence of diffusion, this problem is somewhat mollified, because a balance eventually obtains between the continual regeneration of the finest spatial scales by the flow and their removal by diffusion [10]. Nevertheless, adequate resolution remains a significant issue.

Since accurate two-dimensional simulations are computationally expensive, various reduced descriptions are routinely used to model the progress of the reactions $[1,2,11,12]$. The crudest of these ignore the segregation entirely, and model the system as becoming instantaneously well mixed [1]. Such approximations are appropriate when the Péclet number $\mathcal{P}$, the ratio of diffusive to advective time scales, is small. A more sophisticated class of models proceeds from the observation that chaotic fluid mixing generates a complex array of intertwined striations, which, while exceedingly intricate, are well approximated over significant portions of the flow domain as arrays of parallel lamellae. It is then reasonable to replace the two-dimensional problem by a one-dimensional problem for reactions taking place in a lamellar array, allowing significant analytical and numerical progress [13-21]. In this paper, we compare full twodimensional simulations with corresponding one-dimensional simulations of lamellar models (of varying degrees of sophistication), to evaluate the lat- 
ter. It is not a priori obvious how quantitatively accurate one should expect the lamellar models to be, given that they generally ignore many details of the striations generated by the flow, for instance: the curvature of the striations $[22,23]$, the nonuniformity of the stretching [24], the nonuniformity of the striation widths [16], the presence of islands in the flow [6,9], and the continual regeneration of striations (by fluid mechanical stretching and folding, which lead to more, thinner striations, which then recombine diffusively). One would not be surprised to find that they perform well at low Péclet numbers, where diffusion rapidly homogenises the reactant distribution, and poorly at high Péclet numbers, where the flow details cited above become more significant. The present paper aims to provide quantitative background to such intuition.

The flow adopted here is the well studied sine flow [25,26], which comprises alternating shear flows in orthogonal directions. For an appropriate choice of its parameters, the sine flow readily generates a chaotic flow with only very small islands of regular motion. Although this flow is somewhat artificial, it combines the stretching and folding mechanisms of other more realistic chaotic fluid flows, and permits a particularly convenient numerical implementation $[10,27,28]$ of the governing advection-reaction-diffusion equations. For these reasons, it is chosen as the basis for our computational work.

Numerical simulations of the two-dimensional problem tackled here have previously been carried out by Muzzio and Liu [6] but in the special case of an infinitely fast primary reaction, rather than for the finite reaction rates contemplated here (cf. [9], where finite reaction rates are considered, but for a parallel-competitive reaction scheme). These simulations [6] have demonstrated the influence of the mixing on the yield: in general a chaotic fluid flow contains regions of both regular and chaotic motion, and each affect the progress of the reactions in different ways. The regular regions act as poorly mixed reservoirs for the reactants, allowing only a slow leakage out into the chaotic region, through diffusion. In the chaotic region, by contrast, the mixing is rapid. When diffusion is relatively weak (large $\mathcal{P}$ ), the effects of the fluid mixing upon the reaction are most marked. However, as pointed out by Adrover, Cerbelli and Giona [27], the finite-difference space discretisation used by Muzzio and Liu [6] introduces significant spurious numerical diffusion, and its results are unreliable precisely in this limit, over a range of large Péclet numbers reported. The pseudospectral approach adopted here is free from such problems - the advantages are quantified by Adrover, Cerbelli and 
Giona [27], who explicitly demonstrate the errors in large- $\mathcal{P}$ finite-difference simulations when compared with corresponding spectral simulations.

The structure of this paper is as follows. In $\S 2$ we set up the mathematical formulation of the two-dimensional advection-reaction-diffusion problem to be tackled. In $\S 3$ we describe the numerical method for solving the governing equations, and present some numerical results. In $\S 4$ we present a variety of reduced models, and compare their results with corresponding results from the full two-dimensional problem. We close that section with a discussion of the extent to which the present results are surprising. Our conclusions are drawn in $\S 5$.

\section{Mathematical formulation}

We consider the evolution of the two-stage competitive-consecutive reaction [1]

$$
A+B \stackrel{K_{1}}{\longrightarrow} R, \quad B+R \stackrel{K_{2}}{\longrightarrow} S,
$$

from an initial state in which the reactants $A$ and $B$ are completely segregated and the products $R$ and $S$ (which represent, respectively, the desired product and waste) are absent. The reaction takes place in a liquid phase, and a twodimensional chaotic fluid motion stirs the reaction. It is straightforward to see from (1) that in regions where $A$ is locally in excess the fractional yield of $R$ will be greater than in regions where $B$ is locally in excess, since in the latter case the relative abundance of $B$ will promote the secondary reaction $[1,29]$. Details of the way in which the reactants are introduced to one another (i.e., details of the mixing) thus affect the yield of the desired product $R$, and it is this effect that we investigate in this paper. The four chemical species are assumed to undergo Fickian diffusion, and to be passively advected with the flow, i.e., the presence of reactants does not influence the flow, and all species remain in the liquid phase (so there is no precipitation, for instance). The chemical species are modelled as continua, rather than through, say, discrete Monte Carlo simulation (cf. [5]).

In deriving our mathematical formulation of the problem, we suppose that the flow domain is a square of side $L$ and the maximum fluid speed is $U$. We denote a typical reactant concentration by $C$, and assume that all chemical 
species have equal diffusivity, $D$ (equality is by no means essential for our numerical work, although it greatly simplifies some of the associated analysis). We then nondimensionalise the standard advection-reaction-diffusion equations using $L, U, L / U$ and $C$ as scales for length, velocity, time and chemical concentration, respectively. The resulting governing equations are

$$
\begin{aligned}
& \frac{\partial A}{\partial t}+\boldsymbol{u} \cdot \nabla A=\mathcal{P}^{-1} \nabla^{2} A-k_{1} A B, \\
& \frac{\partial B}{\partial t}+\boldsymbol{u} \cdot \nabla B=\mathcal{P}^{-1} \nabla^{2} B-k_{1} A B-k_{2} B R, \\
& \frac{\partial R}{\partial t}+\boldsymbol{u} \cdot \nabla R=\mathcal{P}^{-1} \nabla^{2} R+k_{1} A B-k_{2} B R, \\
& \frac{\partial S}{\partial t}+\boldsymbol{u} \cdot \nabla S=\mathcal{P}^{-1} \nabla^{2} S+k_{2} B R,
\end{aligned}
$$

where $A, B, R$ and $S$ denote the corresponding dimensionless chemical concentrations and $\boldsymbol{u}=(u, v)$ is the dimensionless velocity field, specified below. The dimensionless parameters present here are the Péclet number $\mathcal{P}=U L / D$ and two dimensionless reaction rates, $k_{1}=K_{1} C L / U$ and $k_{2}=K_{2} C L / U$. Note that each of $k_{1}$ and $k_{2}$ takes the form of the ratio of a second Damköhler number to the Péclet number [6].

The initial conditions are of segregation, with

$$
\begin{aligned}
& A(x, y, 0)= \begin{cases}A_{0} & 0 \leq x<\frac{1}{2}, \\
0 & \frac{1}{2} \leq x<1,\end{cases} \\
& B(x, y, 0)=\left\{\begin{array}{cc}
0 & 0 \leq x<\frac{1}{2}, \\
B_{0} & \frac{1}{2} \leq x<1,
\end{array}\right.
\end{aligned}
$$

and with neither product initially present: $R(x, y, 0)=S(x, y, 0)=0$. To avoid the eventual consumption of all the desired product $R$ in the secondary reaction, we must take $A_{0} / B_{0}>1 / 2$.

The fluid motion takes place in a (dimensionless) square box given by $0 \leq x \leq$ $1,0 \leq y \leq 1$. The sides $x=0$ and $x=1$ are identified with one another, so that fluid escaping from the unit square through one reenters through the other (and similarly for the sides $y=0$ and $y=1$ ). The flow is two-dimensional and incompressible, and comprises a time-periodic shear flow, alternately parallel 

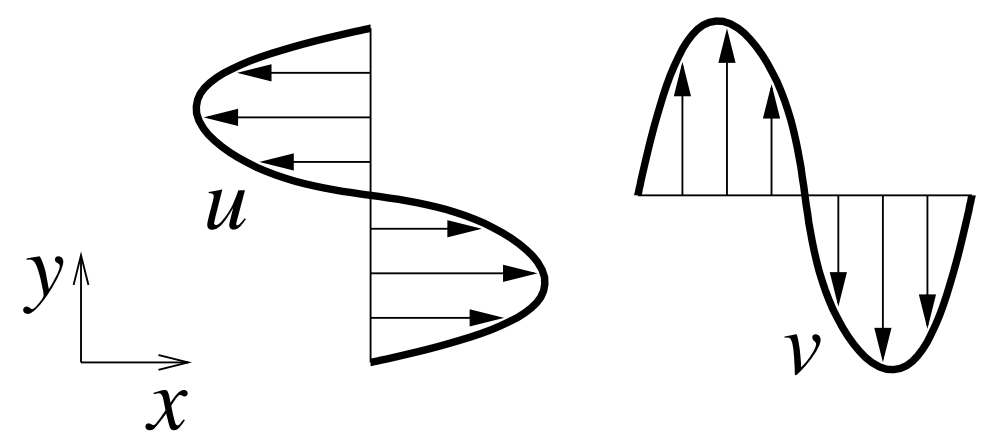

Fig. 1. Sine flow (8), (9). Left: flow during first half-period. Right: flow during second half-period.

to the $x$ - and $y$-axes. Denoting the period of the motion by $T$, we define the velocity components to be

$$
\begin{aligned}
& u=\left\{\begin{array}{cl}
\sin 2 \pi y & \nu T \leq t<\left(\nu+\frac{1}{2}\right) T, \\
0 & \left(\nu+\frac{1}{2}\right) T \leq t<(\nu+1) T,
\end{array}\right. \\
& v=\left\{\begin{array}{cl}
0 & \nu T \leq t<\left(\nu+\frac{1}{2}\right) T, \\
\sin 2 \pi x & \left(\nu+\frac{1}{2}\right) T \leq t<(\nu+1) T,
\end{array}\right.
\end{aligned}
$$

for $\nu=0,1,2, \ldots[25,26]$ - see Figure 1 . For general values of $T$, the flow domain contains islands of periodic or quasiperiodic motion surrounded by a region of chaotic motion. We examine two cases: $T=0.8$, in which the majority of the flow domain is chaotic, but with four significant regions of regular behaviour, and $T=1.6$, in which the motion is predominantly chaotic, and for which it is seen, by an examination of the Poincaré map, that any regular islands are so small that they are indiscernible to the eye - see Figure 2 for corresponding Poincaré maps. By investigating these two cases, we gain some insight into the large-time influence of large islands $(T=0.8)$ on the mixing compared with - to the eye, at least - a 'globally chaotic' flow $(T=1.6)$. 

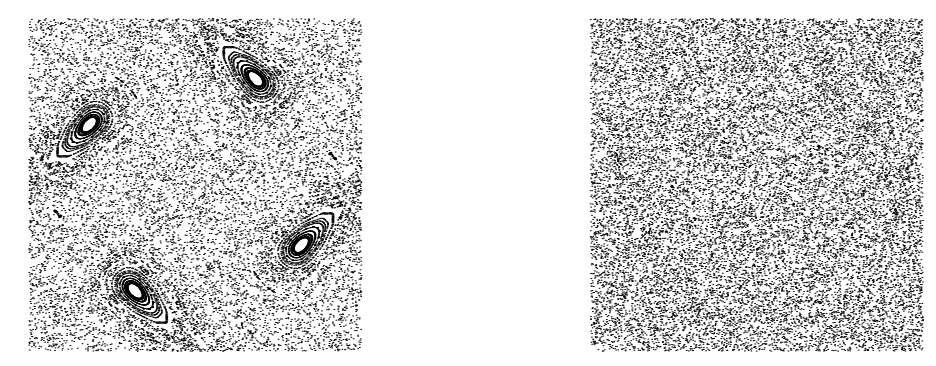

Fig. 2. Poincaré maps for the sine flow with $T=0.8$ (left) and $T=1.6$ (right).

\section{Two-dimensional numerical simulations}

\subsection{Numerical method}

The governing equations (2)-(5) are solved using a pseudospectral method $[10,27,28,30]$, which permits simulations of high accuracy, even at large $\mathcal{P}$, principally because it does not suffer from the spurious numerical diffusion associated with alternative finite-difference or finite-element calculations [27].

In our implementation, we expand each dependent variable as a Fourier series (truncated for numerical purposes): for example,

$$
A(x, y, t) \sim \sum_{m=-N}^{N} \sum_{n=-N}^{N} A_{m n}(t) \mathrm{e}^{2 \pi \mathrm{i}(m x+n y)} .
$$

These Fourier expansions are then substituted into the governing equations (2)-(5) to yield a system of ordinary differential equations in time for $A_{m n}(t)$, $B_{m n}(t), R_{m n}(t)$ and $S_{m n}(t)$. To ensure that the code runs quickly, the nonlinear reaction terms are computed in physical space, the transformations between spectral and physical space being achieved through the Fast Fourier Transform. The system of ordinary differential equations is stiff, and we use exponential time-differencing [31] to solve it, specifically the second-order RungeKutta method ETD2RK [32], since this method permits high accuracy even with relatively large time steps.

To describe our implementation of exponential time-differencing, we note that the equation for $A_{m n}$, for instance, takes the form

$$
\frac{\mathrm{d} A_{m n}}{\mathrm{~d} t}=-4 \pi^{2} \mathcal{P}^{-1}\left(m^{2}+n^{2}\right) A_{m n}-\pi m\left(A_{m(n-1)}-A_{m(n+1)}\right)+N_{m n}^{A}
$$


for $\nu T \leq t<\left(\nu+\frac{1}{2}\right) T$, where the terms on the right-hand side represent, in order, diffusion, advection and reaction. A corresponding expression holds during the other half-period, but with the advection term in (10) replaced by $-\pi n\left(A_{(m-1) n}-A_{(m+1) n}\right)$. The stiffness of the system comprising (10) and the corresponding equations for the other chemical species is evident from the diffusion term, which generates widely differing time scales for the evolution of the various modes - in particular, there is very rapid diffusive decay of the high-wavenumber modes. The problems associated with linear stiffness are obviated in the exponential time-differencing method because it treats the linear terms exactly, the only approximation lying with the integration of the nonlinear terms. Although alternative implementations are certainly possible, we find it convenient to combine the advection and reaction terms, and treat these as being 'nonlinear', with only the diffusion term treated as 'linear', and hence integrated exactly. Thus we write (10) as

$$
\frac{\mathrm{d} A_{m n}}{\mathrm{~d} t}=-4 \pi^{2} \mathcal{P}^{-1}\left(m^{2}+n^{2}\right) A_{m n}+\mathcal{N}_{m n}^{A}
$$

where the definition of $\mathcal{N}_{m n}^{A}$ follows by comparison of (10) with (11).

We now denote by $A_{m n}^{l}$ the mode amplitude $A_{m n}$ at $t=l h$, where $h$ is the time step. To execute a single time step using the ETD2RK scheme [32], we first compute

$$
\bar{A}_{m n}^{l}=\mathrm{e}^{c h} A_{m n}^{l}+\frac{\mathrm{e}^{c h}-1}{c} \mathcal{N}_{m n}^{l}
$$

(and, correspondingly, $\bar{B}_{m n}^{l}, \bar{R}_{m n}^{l}$ and $\bar{S}_{m n}^{l}$ ) and then complete the time-stepping through

$$
A_{m n}^{l+1}=\bar{A}_{m n}^{l}+\frac{\mathrm{e}^{c h}-1-c h}{c^{2} h}\left(\overline{\mathcal{N}}_{m n}^{l}-\mathcal{N}_{m n}^{l}\right)
$$

where $c=-4 \pi^{2}\left(m^{2}+n^{2}\right) / \mathcal{P}$, and where the advection-reaction term $\overline{\mathcal{N}}_{m n}^{l}$ is evaluated using $\bar{A}_{m n}^{l}, \bar{B}_{m n}^{l}, \bar{R}_{m n}^{l}$ and $\bar{S}_{m n}^{l}$. The scheme (12), (13) is applied to all modes except those for which $m=n=0$, where we instead use the second-order standard Runge-Kutta scheme

$$
\begin{aligned}
\bar{A}_{00}^{l} & =A_{00}^{l}+h \mathcal{N}_{00}^{l} \\
A_{00}^{l+1} & =\bar{A}_{00}^{l}+\frac{1}{2} h\left(\overline{\mathcal{N}}_{00}^{l}-\mathcal{N}_{00}^{l}\right) .
\end{aligned}
$$



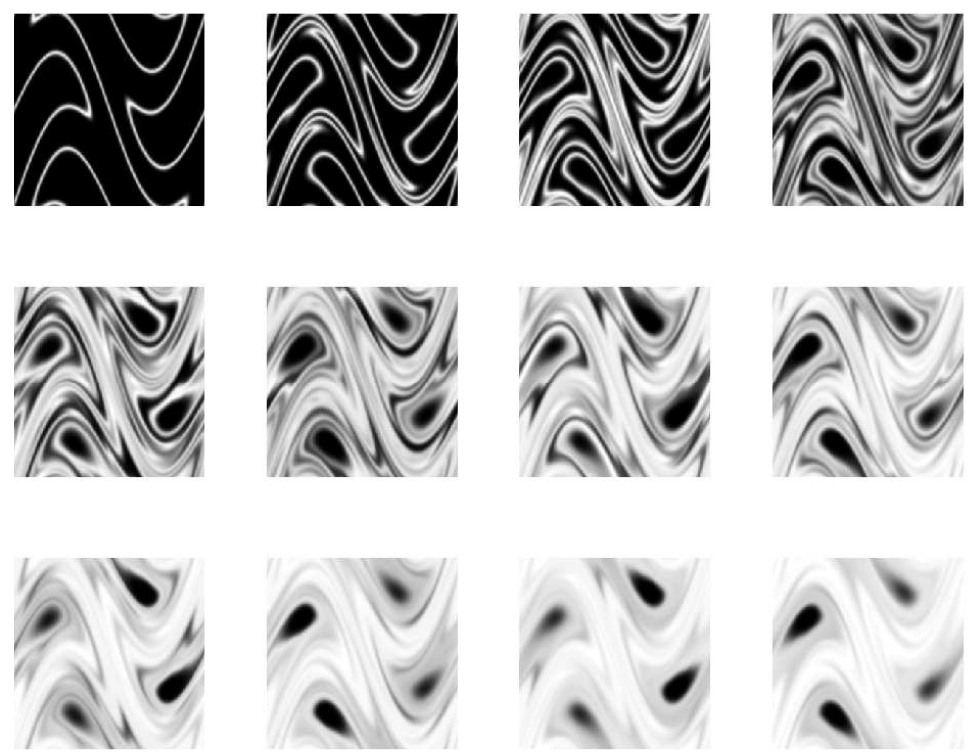

Fig. 3. Concentration field of $R$ after $\nu$ periods of the sine flow for $\nu=1,2, \ldots, 12$ (reading along rows, from top left to bottom right) at $T=0.8$ and $\mathcal{P}=10^{4}$.
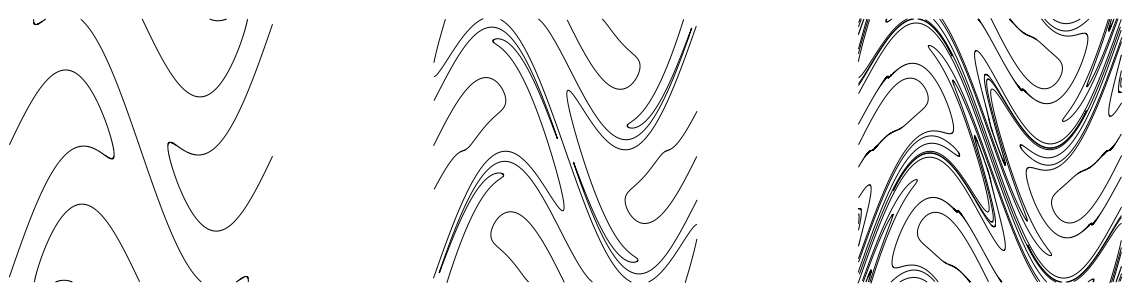

Fig. 4. Kinematic evolution of the interfaces $x=0$ and $x=1 / 2$ after, from left to right, one, two and three periods of the sine flow $(T=0.8)-\mathrm{cf}$. the first three plots of Figure 3.

We choose $N$ and $h$ to obtain solutions that have converged to the precision quoted. The adequacy of the resolution in time and space is checked by repeating sample runs at a smaller time step or with more Fourier modes. We have also noted the quantitative results on the accuracy of spectral schemes obtained for the case $k_{2}=0$ by Adrover, Cerbelli and Giona [27].

\subsection{Numerical results}

Numerical simulations of (2)-(5) subject to (6)-(9) are now presented. In most simulations a resolution of $N=256$ and time step of $h=1.6 \times 10^{-4}$ proved adequate. In all simulations we take $A_{0}=B_{0}=2$. We describe in this section detailed, largely qualitative, results for $\mathcal{P}=10^{4}$ and for 'fast' reactions, with 
$k_{1}=10$ and $k_{2}=1$ (thus the secondary reaction is an order of magnitude slower than the primary reaction). This choice of parameters ensures that the fluid mechanical mixing has a significant influence upon the yield of the reaction - the simulations presented here represent a compromise between studying large $\mathcal{P}$, where the effects of mixing are most marked, and numerical manageability. Summary quantitative results are presented later in $\S 4.4$ for a wide range of other Péclet numbers.

\subsection{1 $T=0.8$}

Figure 3 shows grey-scale plots of the concentration of $R$ after $1,2, \ldots, 12$ periods of the sine flow with $T=0.8$ and $\mathcal{P}=10^{4}$. In each plot, black corresponds to zero concentration and white to the maximum concentration in that plot; thus the scale changes between plots. However, the figure is little altered by adopting the same grey-scale for all plots, because the minimum concentration of $R$ remains close to zero (in two of the islands, in later plots) and the maximum concentration of $R$ varies by less than $10 \%$ between plots. Initially, $R$ is produced in a thin region around material lines whose initial locations are the interfaces $x=0$ and $x=1 / 2$ between the reactants $A$ and $B$, and which are passively advected by the flow: the evolution of such material lines after one, two and three periods of the flow is shown in Figure 4 for comparison. The rapid generation of a complicated pattern of striations of $R$ can clearly be seen in Figure 3, as can the emergence of four islands of less well mixed fluid, corresponding to the islands of regular motion in Figure 2. Also apparent is the rapid merging of the finest spatial scales through diffusion. This is particularly evident when the first three plots in Figure 3 are compared with corresponding plots in Figure 4 (diffusion being absent in the latter figure).

The markedly different behaviour in the chaotic and regular regions is evident in the later plots of Figure 3. In the chaotic region, the chemical species are essentially homogeneously distributed in space through the repeated kinematic generation of small scales and their subsequent smoothing by diffusion. Furthermore, after 12 periods the reaction is essentially complete - the total yield $\langle R\rangle \equiv \int_{0}^{1} \int_{0}^{1} R(x, y, t) \mathrm{d} y \mathrm{~d} x$ has reached $99.3 \%$ of its ultimate value, even while four islands of poor mixing remain. Of the four islands shown after 12 periods of the flow, the left-hand pair contain significant amounts of $S$ (they were

initially $B$-rich); the right-hand pair contain significant amounts of $R$ (they 

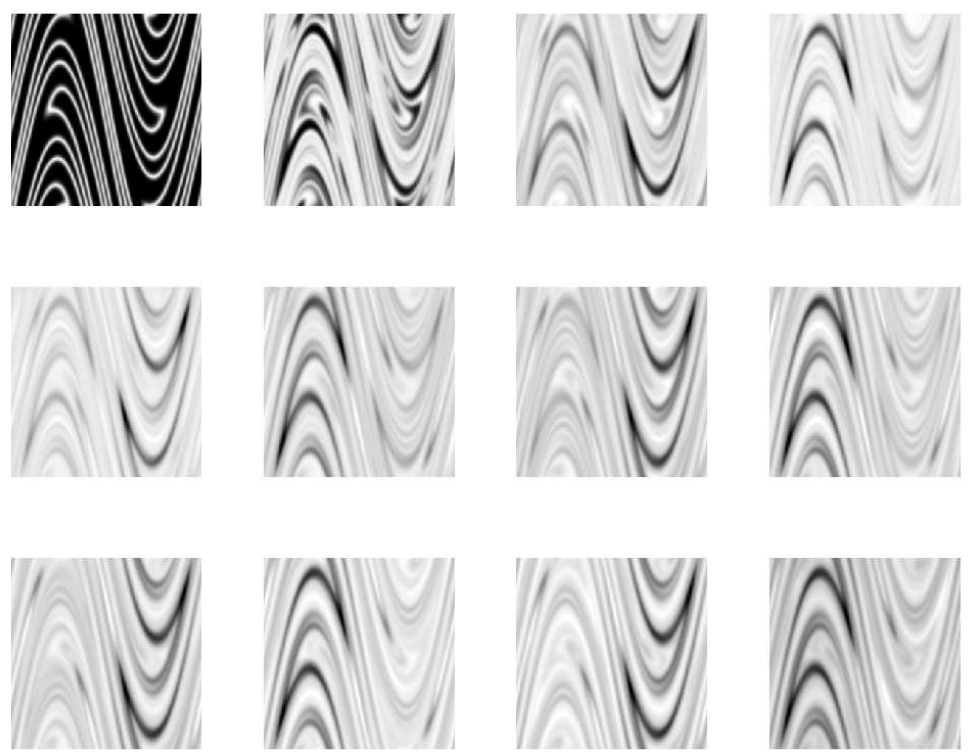

Fig. 5. Concentration field of $R$ after $1,2, \ldots, 12$ periods of the sine flow (reading along rows, from top left to bottom right) at $T=1.6$ and $\mathcal{P}=10^{4}$. In each plot, white and black correspond, respectively, to maximum and minimum concentrations in that plot.

were initially $A$-rich). The mechanism by which these islands of inhomogeneity are ultimately removed is primarily through slow diffusive leakage across the boundary between regular and chaotic regions. Note, from Figure 3, that the concentration field of $R$ (and of the other chemical species - not shown) settles to a persistent oscillation at twice the period of the sine flow itself (cf. [33]).

\subsection{2 $T=1.6$}

When $T=1.6$, the sine flow generates a fluid motion containing no discernible regular islands (by which we mean that none are clearly visible in a Poincaré map - see Figure 2). Corresponding concentration fields of $R$ and $S$ are shown, again for $\mathcal{P}=10^{4}$, in Figures 5 and 6 , respectively. In contrast to the case $T=0.8$, these figures look rather different if all sub-plots are given the same concentration scale - see Figure 7. Again, note the persistent period-two oscillations that become established in the concentration fields. As Figure 8 indicates, the evolution of $\langle R\rangle$ is identical to the previous case $(T=0.8)$ until the first 'flip' of the velocity field (which takes place at $t=0.4$ in the case $T=0.8$ ). Thereafter two significant differences between the yield with $T=0.8$ and $T=1.6$ are apparent: first, that the rate of production of $R$ is enhanced, and second that the ultimate yield is greater in the more globally 

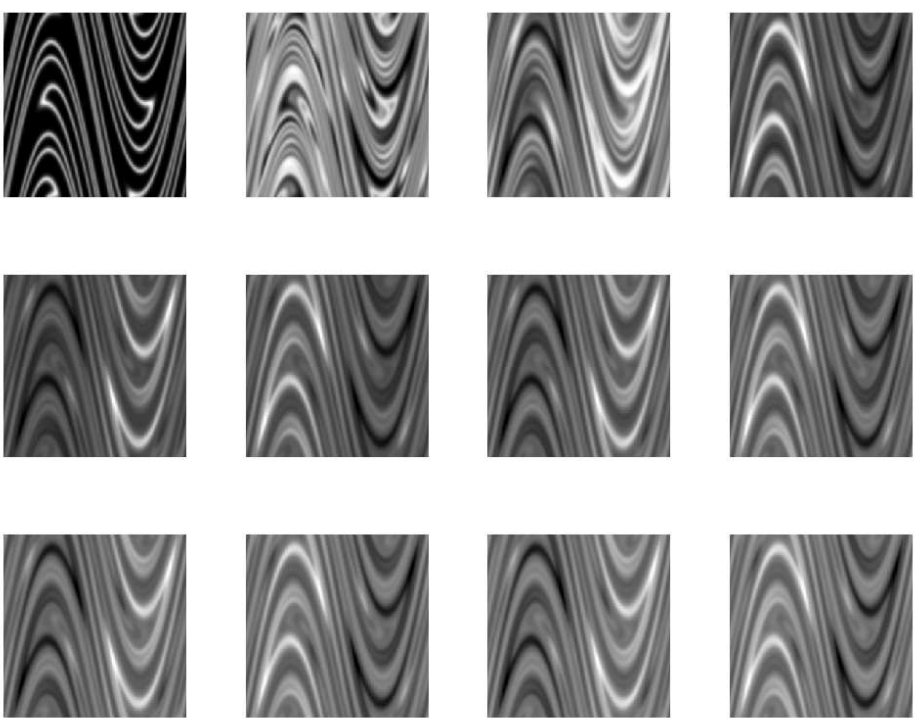

Fig. 6. Concentration field of $\mathrm{S}$ after $1,2, \ldots, 12$ periods of the sine flow (reading along rows, from top left to bottom right) at $T=1.6$ and $\mathcal{P}=10^{4}$. In each plot, white and black correspond, respectively, to maximum and minimum concentrations in that plot.
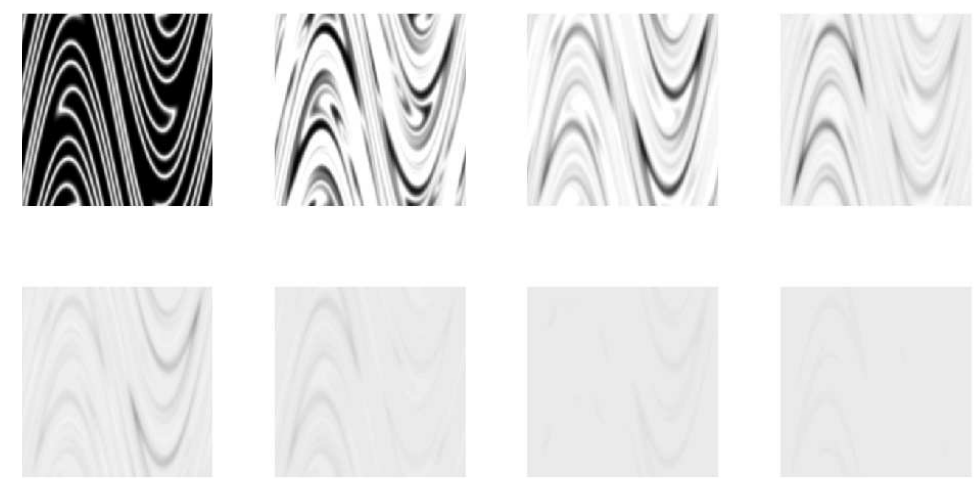

Fig. 7. As first two rows of Figure 5, except that all plots have common grey-scale, with black corresponding to zero concentration and white to the concentration at large time. Note the loss of contrast with time.

chaotic flow. The curve of $\langle R\rangle$ against $t$ is also rather smoother for $T=1.6$ than for $T=0.8$ (this is more obvious when the data are plotted on a linear rather than a logarithmic scale), since in the former case the reactants are more smoothly distributed, while in the latter there are slow events associated with diffusion from the persistent regular regions. 


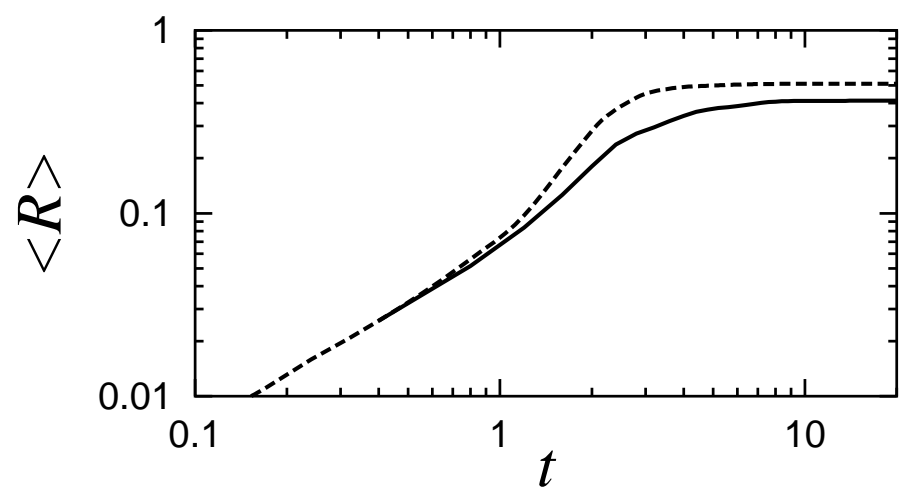

Fig. 8. Plot of total amount $\langle R\rangle$ against $t$ for $\mathcal{P}=10^{4}$. Upper and lower curves correspond, respectively, to $T=1.6$ and $T=0.8$.

\section{Reduced models}

In this section we describe various reduced models of the full two-dimensional system in the 'fully chaotic' regime with $T=1.6$; their success in describing the behaviour of the full system will be evaluated in $\S 4$.4. Our aim is not to develop the most sophisticated possible versions of these models, but to illustrate the type of models that might commonly be used to describe reaction in laminar mixing flows. Not all of these models were developed for application in the present context. The first model, described in $\S 4.1$, assumes that the reactants are instantaneously perfectly mixed by the flow, and that the reaction then proceeds in a spatially homogeneous fashion. The second, described in $\S 4.2$, follows the evolution of the reaction at an interface; this interface remains planar, and does not feel the effects of the chaotic 'folding', although the effects of fluid-mechanical stretching may be modelled. The final models, in $\S 4.3$, describe the evolution of the reaction in a one-dimensional system of lamellae.

\subsection{Well mixed model}

Perhaps the crudest model for the evolving chemical reaction involves ignoring completely the segregation, and considering instead the much simpler spatially uniform problem [1]. One might expect such an approximation to apply when diffusion acts rapidly to homogenise the initially segregated state, i.e., in the limit $\mathcal{P} \rightarrow 0$ (for fixed $k_{1}$ and $k_{2}$ ). 
The well mixed model thus comprises the ordinary differential equations obtained by omitting spatial derivatives from (2)-(5) together with initial conditions of spatially uniform concentrations $A=\frac{1}{2} A_{0}, B=\frac{1}{2} B_{0}, R=S=0$. (The factors of $\frac{1}{2}$ reflect the mean initial concentrations of $A$ and $B$ corresponding to $(6),(7)$.) This system can readily be reduced to a single ordinary differential equation for $A(t)$ (see, for example, [1,15]), and the large-time state of the system then deduced to be as follows. If $A_{0} / B_{0}<1 / 2$, all of $A$ is consumed and it is a simple matter to determine the ultimate state of the system in terms of the initial stoichiometry: at large times $A \rightarrow 0, B \rightarrow \frac{1}{2} B_{0}-A_{0}, R \rightarrow 0$ and $S \rightarrow \frac{1}{2} A_{0}$. If $A_{0} / B_{0}>1 / 2$, it is necessary to consider the time evolution of the reactants in order to determine the final state; this case is of more practical interest than the previous one because some of the desired product remains at large time: in this limit $A \rightarrow \frac{1}{2} A_{\infty}, B \rightarrow 0, R \rightarrow A_{0}-\frac{1}{2} B_{0}-A_{\infty}$ and $S \rightarrow \frac{1}{2}\left(B_{0}-A_{0}+A_{\infty}\right)$, where $A_{\infty}$ satisfies [1], for $k_{2} \neq k_{1}$,

$$
\left(1-2 k_{2} / k_{1}\right) A_{\infty} / A_{0}+\left(A_{\infty} / A_{0}\right)^{k_{2} / k_{1}}=\left(1-k_{2} / k_{1}\right)\left(2-B_{0} / A_{0}\right)
$$

A corresponding result for $k_{2}=k_{1}$ is given by Levenspiel [1], but it is not of direct interest here, since we assume that the reaction scheme has been designed so that the secondary reaction producing waste has a smaller rate constant than the primary reaction. In the special case $k_{2}=k_{1} / 2,(16)$ is readily solved to give the explicit solution $A_{\infty}=\frac{1}{4}\left(2 A_{0}-B_{0}\right)^{2} / A_{0}$, but otherwise it possesses no straightforward analytical solution, and requires numerical solution.

\subsection{Single, planar interface}

Clearly, in view of the influence of reactant segregation upon yield, it is desirable to adopt a more sophisticated approach than the well mixed model, as demonstrated explicitly in our evaluations in $§ 4.4$. A first step in modelling the advection, reaction and diffusion that takes place in an initially segregated two-dimensional system is to consider these processes in one space dimension [34-39], corresponding to ignoring the kinematic distortion of the interfaces $x=0$ and $x=\frac{1}{2}$ under the flow. In this model, the reactants $A$ and $B$ initially lie on either side of a single infinite planar interface. Although such a one-dimensional model clearly cannot account entirely for the effects of fluid mechanical mixing, the exponential stretching of material line elements under 
the flow may be mimicked by imagining the one-dimensional problem to take place in a straining flow. We shall take a simple, commonly adopted model for this straining flow (although a more sophisticated approach is certainly feasible). This single-interface model might be expected to be valid at sufficiently early times [2], before neighbouring interfaces influence one another, and before curvature of the interface is significant.

In this model, the reactants $A$ and $B$ lie initially on either side of $x=0$; all chemical concentrations remain independent of the $y$-coordinate for all time. The chemical species diffuse (in the $x$-direction) and react; they are also advected by a straining flow $(u, v)=(-\mu x, \mu y)$, which allows us to retain the effect of mixing in stretching the interface exponentially in time, but without the corresponding folding $[2,12,13,16-18,40-42]$. The appropriate dimensionless governing equations are then

$$
\begin{aligned}
A_{t}-\mu x A_{x} & =\mathcal{P}^{-1} A_{x x}-k_{1} A B, \\
B_{t}-\mu x B_{x} & =\mathcal{P}^{-1} B_{x x}-k_{1} A B-k_{2} B R, \\
R_{t}-\mu x R_{x} & =\mathcal{P}^{-1} R_{x x}+k_{1} A B-k_{2} B R, \\
S_{t}-\mu x S_{x} & =\mathcal{P}^{-1} S_{x x}+k_{1} B R,
\end{aligned}
$$

for $-\infty<x<\infty$, where $\mu>0$ characterises the rate of stretching of the interface. It is debatable what value should be adopted for $\mu$ in the model, for a number of reasons. First, there are two obvious candidates: an (averaged) Lyapunov exponent, which represents local stretching, and the topological entropy, which represents the growth rate of finite material lines, and which generally exceeds the Lyapunov exponent [43-45]. Second, the distribution of stretch rates along any material line is nonuniform [24], which causes difficulties in choosing a single value for $\mu$. Third, it is not clear that exponential (rather than linear) growth of the interface is appropriate at early times, when this model is intended to be valid [46]. We note that in any case it is common to investigate the effects of segregation separately from those of mixing, i.e., most attention has been addressed to the special case $\mu=0$, where the question of the correct value of $\mu$ does not arise [11,14-16,18-21,35-39,47-49].

The appropriate initial conditions are of segregated reactants in the following form: for $x<0, A(x, 0)=A_{0}$ and $B(x, 0)=0$; for $x>0, A(x, 0)=0$ and $B(x, 0)=B_{0}$; for all $x, R(x, 0)=S(x, 0)=0$. The boundary conditions are: as $x \rightarrow-\infty, A \rightarrow A_{0}$ and $B, R, S \rightarrow 0$; as $x \rightarrow \infty, B \rightarrow B_{0}$ and $A, R, S \rightarrow 0$. 
Although some analytical progress is possible for chemical species having different relative rates of diffusion $[35,37,38,48,49]$, greatest progress can be achieved when, as here, all four species have identical diffusivities. Then it follows immediately by taking linear combinations of (17)-(20) that the quantities

$$
\alpha=2 A-B+R, \quad \beta=A+R+S
$$

satisfy the linear equations $\alpha_{t}-\mu x \alpha_{x}=\mathcal{P}^{-1} \alpha_{x x}$ and $\beta_{t}-\mu x \beta_{x}=\mathcal{P}^{-1} \beta_{x x}$, which are readily solved (see below) given the initial and boundary conditions appropriate here. For analysis of (17)-(20), it is useful to introduce the new independent variables $X=x \mathrm{e}^{\mu t}$ and $T=\frac{1}{2} \mathcal{P}^{-1}\left(\mathrm{e}^{2 \mu t}-1\right) / \mu$, corresponding to the 'warped time transformation' of Ranz [12] (cf. [34], Eqs (2) and (3)). Under this transformation, the problem for the evolution of $\alpha$ and $\beta$ simplifies to $\alpha_{T}=\alpha_{X X}$ and $\beta_{T}=\beta_{X X}$. These equations are readily solved subject to the relevant initial and boundary conditions to give

$$
\alpha=A_{0}(1-\operatorname{erf} \eta)-\frac{1}{2} B_{0}(1+\operatorname{erf} \eta), \quad \beta=\frac{1}{2} A_{0}(1-\operatorname{erf} \eta)
$$

where $\eta=\frac{1}{2} X T^{-1 / 2}$, and these solutions apply for $T>0$ (i.e., for $t>0$ ). Once $\alpha$ and $\beta$ are found, $A$ and $B$ may then be determined by solving

$$
\begin{aligned}
& A_{T}=A_{X X}-\frac{\mathcal{P}}{2 \mathcal{P} \mu T+1} k_{1} A B \\
& B_{T}=B_{X X}-\frac{\mathcal{P}}{2 \mathcal{P} \mu T+1}\left[\left(k_{1}-2 k_{2}\right) A B+k_{2}(\alpha+B) B\right]
\end{aligned}
$$

the concentrations of $R$ and $S$ are then recovered from (21).

\subsubsection{Early time}

The present single-interface model should hold most accurately at early time, and so we now focus on this limit. In order to compute, for instance, the yield of $R$ at early time, it is useful to reformulate (23), (24) in terms of the independent variables $\eta$ and $T$, giving

$$
A_{\eta \eta}+2 \eta A_{\eta}-4 T A_{T}=\frac{4 \mathcal{P} T}{2 \mathcal{P} \mu T+1} k_{1} A B
$$




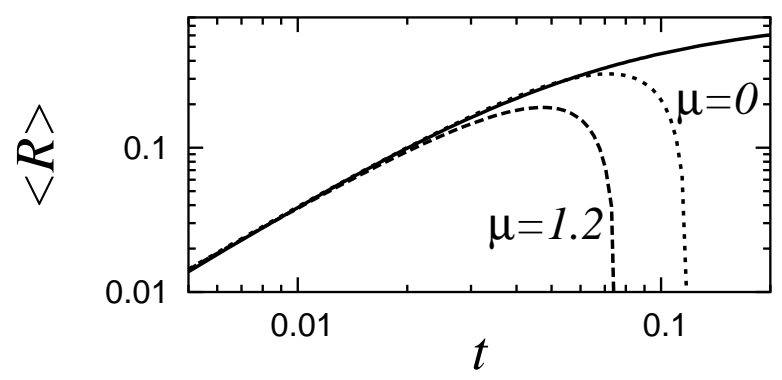

Fig. 9. Plot showing agreement between early-time prediction of $\langle R\rangle$ (dotted line $\mu=0$; dashed line $\mu=1.2$ ) and value computed from full two-dimensional simulations (solid line) for $T=1.6$ and $\mathcal{P}=1$.

$$
B_{\eta \eta}+2 \eta B_{\eta}-4 T B_{T}=\frac{4 \mathcal{P} T}{2 \mathcal{P} \mu T+1}\left(k_{1}-2 k_{2}\right) A B+\frac{4 \mathcal{P} T}{2 \mathcal{P} \mu T+1} k_{2}(\alpha+B(2 B)
$$

At early time, we may expand the solution to (25), (26) in powers of $T$ as $A \sim \hat{A}_{0}(\eta)+T \hat{A}_{1}(\eta)+\cdots$ and $B \sim \hat{B}_{0}(\eta)+T \hat{B}_{1}(\eta)+\cdots$, with the corresponding expansion for the product $R$ being $R \sim T \hat{R}_{1}(\eta)+T^{2} \hat{R}_{2}(\eta)+\cdots$ (cf. $[11,15,36]$ ). Since we have elsewhere presented the early-time analysis in the absence of fluid mixing [15] (i.e., for $\mu=0$ ), we relegate a sketch of the calculation to the Appendix, highlighting those parts that are required for a computation of the mixing effects. Such effects are found by continuing the expansion for $R$ to $O\left(T^{2}\right)$, from which we deduce the total amount of $R$ produced, per unit initial length in $y$, to be

$$
\begin{aligned}
\langle R\rangle & \equiv \mathrm{e}^{\mu t} \int_{-\infty}^{\infty} R \mathrm{~d} x \\
& \sim 2 k_{1} \mathcal{P} A_{0} B_{0} I T^{1 / 2}\left\{\frac{1}{6} T-\mathcal{P} T^{2}\left[\frac{1}{30}\left(k_{1} A_{0}+\left(k_{1}+k_{2}\right) B_{0}\right)+\frac{1}{5} \mu\right]\right\}
\end{aligned}
$$

where $I \approx 1.5958$. Thus the fluid mixing $(\mu>0)$ tends to suppress the early net production of $R$, regardless of the initial stoichiometry or relative reaction rates. Note from $(25),(26)$ that this small-time expansion is in powers of $\mathcal{P} T$, and so is of particularly limited utility when $\mathcal{P}$ is large.

In Figure 9 we compare the early-time prediction (27) with the true value obtained from the full two-dimensional simulations (we take $\mu$ to be either zero or the Lyapunov exponent of the sine flow). Inclusion of fluid mixing effects seems to worsen the single planar interface model, in two respects. First, the magnitude of the coefficient of $\mathcal{P} T^{2}$ in (27) is increased, leading to 
divergence of this truncated power series at earlier time. Second, the fit at moderate times seems worse. This result suggests that modelling the effects of the fluid mixing with an exponential normal contraction is inappropriate for the single-interface model (for the times involved in Figure 9, considerably less than one period of the flow, the growth rate of an interface is clearly not exponential).

\subsection{Lamellar models}

A more sophisticated model of the segregation involves not a single interface, but a finite array of lamellae $[2,11]$, to model the striations generated in a chaotic flow such as the sine flow. Most analytical and computational advantage is gained by considering the lamellar array to be one-dimensional $[3,4,11-$ 21,35-42,47-50], although clearly in reality the striations possess significant curvature where they are folded over by the flow, and this curvature can impact on the yield. As with the single-interface model above, the effects of fluid mixing in compressing and stretching the lamellae in (approximately) normal and parallel directions may be considered or not. Lamellar models thus generally resemble the single-interface model described above, except that they take place over a finite extent in $x$ and possess a different initial distribution of reactants. Usually the domain is assumed to be periodic in space, which can lead to considerable simplifications in numerical simulation. As with the two-dimensional simulations described above in $\S 3.2$, we use a pseudospectral method for carrying out the numerical integration of the various onedimensional lamellar models below, again with exponential time-differencing.

There have been many previous studies of the laminar lamellar model. An early analytical and numerical treatment in the absence of fluid mechanical mixing is that of Pearson [11]. Chella and Ottino [4] performed numerical simulation of combined advection, reaction and diffusion in a periodic array of lamellae for a range of reaction schemes, and analysis of various limiting cases (see also [3]). Subsequent calculations [50] for the reaction scheme considered in this paper were facilitated by use of the warped time transformation [12], and show good agreement with the well mixed limit and with experiment, as appropriate (see also [40]). A related set of simulations of the lamellar model for polymerisation was carried out by Fields and Ottino [16-18]. The utility of the lamellar model was demonstrated by Clifford et al. [13], who found reasonable agreement 
with results from two-dimensional simulations of the full advection-reactiondiffusion problem [6], although, as we have already remarked, at large Péclet number the latter simulations are known to be inaccurate [27]. Of particular note are the works of Sokolov and Blumen [41,42], who analysed a single reaction taking place in a lamellar structure, with special emphasis on 'fast' reactions, for which a great deal of analytical progress is possible, and of Muzzio and Ottino $[19,20]$, who examined the evolution of a lamellar structure in which only the primary reaction takes place (i.e., the case $k_{2}=0$ ), at infinite rate. The latter focused on the evolution of the distribution of striation thicknesses, which is not our direct concern here, and commented that it would be interesting to include fluid mechanical mixing effects into their lamellar model. In [21], their work was extended to the case of finite primary reaction rate, this time with a more general form of reaction term than $k_{1} A B$.

Here we adopt a simple form of the lamellar model, which follows the evolution of a single pair of lamellae. A more sophisticated variant of the model represents a more realistic set of lamellae, which have a range of different initial widths matching the striation width distribution measured from full two-dimensional simulations [14,19-21]. This latter approach clearly builds in more information from the full simulations, and might therefore be expected to provide a more accurate lamellar model, but is not considered here. Furthermore, it should be noted that for both of these variants of the lamellar model, the initial distribution of lamellae is 'frozen in', and so neither can accommodate the continual generation of new lamellae (achieved by folding in the two-dimensional flow). It should also be noted that it is not only the lamellar widths that are important: the order in which a given set of lamellae are arranged can also strongly influence the yield [14].

\subsubsection{Lamellar model with continuous stretching}

Our first lamellar model is identical to the single-interface model, except in its initial and boundary conditions, and in the specification of the flow domain. Thus we solve (17)-(20) subject to the initial conditions

$$
A(x, 0)=\left\{\begin{array}{cc}
A_{0} & 0 \leq x<\frac{1}{2} \\
0 & \frac{1}{2} \leq x<1
\end{array}\right.
$$




$$
B(x, 0)=\left\{\begin{array}{cc}
0 & 0 \leq x<\frac{1}{2} \\
B_{0} & \frac{1}{2} \leq x<1
\end{array}\right.
$$

again with $R(x, 0)=S(x, 0)=0$. We assume that the domain shrinks exponentially in the $x$-direction (and correspondingly grows in the $y$-direction with the same exponential factor, $\mu$ ). For numerical purposes it is then most convenient to solve the system as expressed with $X$ and $T$ as the independent variables, since then the domain in $X$ is of fixed size, and we solve

$$
\begin{aligned}
& A_{T}=A_{X X}-\frac{\mathcal{P}}{2 \mathcal{P} \mu T+1} k_{1} A B \\
& B_{T}=B_{X X}-\frac{\mathcal{P}}{2 \mathcal{P} \mu T+1}\left(k_{1} A B+k_{2} B R\right), \\
& R_{T}=R_{X X}-\frac{\mathcal{P}}{2 \mathcal{P} \mu T+1}\left(-k_{1} A B+k_{2} B R\right)
\end{aligned}
$$

for $0 \leq X \leq 1$, with periodic boundary conditions. In evaluating the necessity of including $\mu>0$ to parametrise the fluid mixing, we shall consider below results for both $\mu>0$ and $\mu=0$; these results are presented in $\S 4.4$ below.

\subsubsection{Lamellar model with discrete stretching and 'folding' (baker map)}

As we have indicated above, a potentially significant shortcoming of the usual lamellar models is that although they are able to mimic the stretching aspect of the full two-dimensional chaotic fluid motion, they fail to model the folding back of flow structures upon themselves, which serves continually to regenerate the lamellar structure. Sokolov and Blumen [42] recognised this shortcoming and suggested the use of a baker transformation to regenerate the lamellar structure; such an approach has recently been adopted by Neufeld et al. [7] in the context of an advection-reaction-diffusion system with multiple steady states. In the related lamellar model described below there is continuous reaction and diffusion of chemical species in a one-dimensional lamellar array, subject to periodic episodes of discrete stretching and folding to simulate the corresponding continuous processes in the two-dimensional flow. Clearly there is in reality no such separation between the processes of reaction-diffusion on the one hand and stretching-and-folding on the other, and so it is of particular interest to attempt to evaluate the predictive capabilities of such models (or at least a simplified form of such models, as presented below). 


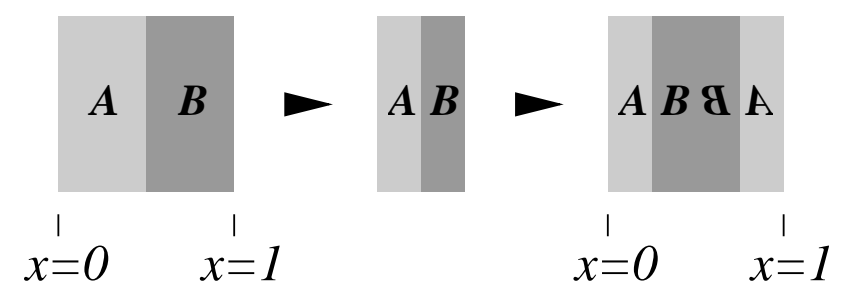

Fig. 10. Application of the baker map to the lamellar array, carried out at discrete times $t=\nu \tau(\nu=1,2,3, \ldots)$. First the lamellae are squashed to half their width, then a mirror image set of lamellae is adjoined along $x=1 / 2$.

The governing equations for the chemical species are thus (17)-(20), subject to the initial conditions (28) and (29), in the flow domain $0 \leq x \leq 1$. Stretching and folding are implemented at times $t=\nu \tau(\nu=1,2,3, \ldots)$ by means of the baker map $[7,42]$ - see Figure 10 - where $\tau$ is determined below to match the rate of stretching in the sine flow. To be explicit, at time $t=n \tau$ we set $A\left(x, n \tau^{+}\right)=A\left(2 x, n \tau^{-}\right)$for $0 \leq x<\frac{1}{2}$, and $A\left(x, n \tau^{+}\right)=A\left(2(1-x), n \tau^{-}\right)$for $\frac{1}{2} \leq x<1$, with corresponding expressions for the other chemical concentrations. Then to determine $\tau$, we note that all fluid elements are stretched by a factor of 2 at each application of the baker map. Thus if a typical stretch rate in the sine flow to be modelled is $\lambda$ then to match the net average stretch of fluid elements, we should take $\tau=(\log 2) / \lambda$.

In our simulations of this model, we shall choose to take $\lambda$ to be the Lyapunov exponent of the sine flow, although it can be argued that it is more appropriate to take instead the (slightly larger) topological entropy [43,44]. Our justification for this choice is that clearly the separation of reaction and diffusion on the one hand, and stretching and folding on the other is itself a crude device, and so the impression of precision in the specification of $\tau=(\log 2) / \lambda$ is illusory; and, of course, many features of the two-dimensional flow are not captured at all by any of our lamellar models (the nonuniformity of stretching in the sine flow; the presence of regular islands; the curvature of the striations, for instance). Note that if we set $\tau=\infty$ then we recover the no-stretch lamellar model from the previous section (i.e., for $\mu=0$ ).

The piecewise implementation of stretch in this model has the consequence that for fast reactions, if only a few stretching periods are required for reaction to go to completion, then the quantised nature of the stretching will be felt, and will tend to reduce the effective applied stretch rate. 


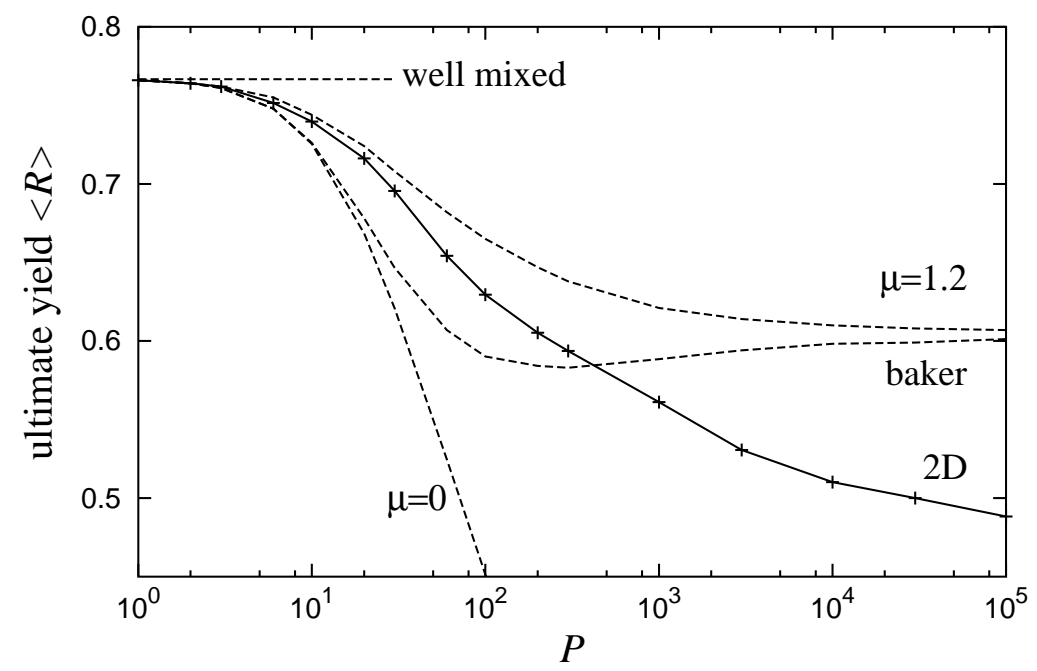

Fig. 11. Final yield of product $R$ as a function of Péclet number $\mathcal{P}$. Curves represent: '2D' full two-dimensional simulations (§3.2); 'well mixed' well mixed model ( $\S 4.1)$; ' $\boldsymbol{\mu}=\mathbf{0}$ ' and ' $\boldsymbol{\mu}=\mathbf{1 . 2}$ ', respectively, lamellar model with no stretch, $\mu=0$, and with stretch rate $\mu=1.2$ (§4.3.1); 'baker' baker map model with $\tau=(\log 2) / 1.2$ $(\S 4.3 .2)$.

\subsection{Comparison between two-dimensional simulations and reduced models}

We have carried out two sets of simulations, corresponding to moderate and fast reaction rates. In the former, we set $k_{1}=1$ and $k_{2}=0.1$, and find the effects of fluid mixing to be comparatively slight, at least over the range $1 \leq \mathcal{P} \leq 10^{5}$ - for example, while the ultimate yield of $R$ appears to be a monotonically decreasing function of $\mathcal{P}$, it falls by less than $8 \%$ as the Péclet number is increased from 1 to $10^{5}$. Therefore, rather than present both sets of results in detail, we focus on the results for fast reactions, where the effects of fluid mixing are more significant, and note briefly corresponding results for moderate reaction rates.

Results for fast reactions $\left(k_{1}=10\right.$ and $\left.k_{2}=1\right)$ are presented in Figure 11, where we show the ultimate yield as a function of $\mathcal{P}$, as computed from our two-dimensional simulations. Accompanying these results are predictions of the yield from the well mixed model and from three variants of the lamellar model. The latter are: the lamellar model with no stretch (i.e., $\mu=0$ ), the continuous-stretch model with $\mu=1.2$ (the Lyapunov exponent) and the baker map model with $\tau=(\log 2) / 1.2$. 
Consider first the limit $\mathcal{P} \rightarrow 0$, corresponding physically to rapid spatial homogenisation through diffusion. In this limit, the results of the well mixed model are approached by all our simulations (i.e., simulations of the twodimensional problem, and of the various one-dimensional lamellar models) - cf. [6,9]. The results from the no-stretch and baker map lamellar models agree extremely closely with one another in this limit. Such agreement is a consequence of the discrete nature of the stretching and folding in the baker map - diffusion is so powerful that by $t=\log 2 / 1.2$ (the first application of the baker map) the homogenisation is essentially complete, and hence the stretching and folding has little effect. We find that the true yield (from the two-dimensional simulations) lies between that obtained from the continuousstretch and baker map lamellar models, and is closer to the former than to the latter.

For $\mathcal{P}$ above around 30, the no-stretch lamellar model is a poor predictor of the yield, although the continuous-stretch and baker map lamellar models remain reasonably accurate (within approximately $7 \%$ ) for $\mathcal{P}$ up to and slightly exceeding 300 . It should be noted that there is a monotonic fall in the yield for both the two-dimensional simulations and the continuous-stretch lamellar model (such a trend agrees with Chella and Ottino [4], although the details are not directly comparable due to the different parameter values in that study and ours), whereas the baker map lamellar model has a (shallow) minimum yield around $\mathcal{P}=200$. We find that for $\mathcal{P}<300$ the yield from the baker map lamellar model is less than that from the full two-dimensional simulation, which in turn is less than that from the continuous-stretch lamellar model. (We find similar trends in our simulations at moderate reaction rate, although the 'threshold' Péclet numbers are approximately three times greater than those given above.)

In the limit of large $\mathcal{P}$, the yields from the continuous-stretch and baker map lamellar models appear to tend towards the same limit: since diffusion is slow in this limit, the reaction proceeds only slowly and many periods are required in order to obtain a significant amount of product. Thus the discrete nature of the mixing in the baker map model is effectively averaged, and mimics well the continuous stretching in the other lamellar model. However, results from the two-dimensional simulations do not appear to approach those of the lamellar models in this large- $\mathcal{P}$ limit. It might thus appear that the lamellar models as we have described them are of limited use in capturing accurately 
the behaviour of the full two-dimensional system. One might attempt to improve the fit of the lamellar models by noting that the no-stretch lamellar model, with $\mu=0$, underestimates the yield (significantly so for $\mathcal{P}$ greater than around 30), while the other lamellar models, with stretch rate $\mu=1.2$ corresponding to a Lyapunov exponent, overestimate the yield. The yield from the lamellar models can therefore be brought into line with the true yield by the device of adopting a reduced value of $\mu$. However, we have found that with such a reduced value of $\mu$ the lamellar models give a poor prediction of the time evolution of the reactants, despite getting the ultimate yield right. It is therefore not clear that the artificial reduction of $\mu$ is appropriate or justified in improving the predictive power of these lamellar models. (Note that the topological entropy is generally greater than the Lyapunov exponent, and we find that using the topological entropy for $\mu$ leads to even worse agreement between lamellar models and full two-dimensional simulations.)

\subsection{Discussion}

One might ask to what extent the present results are surprising: certainly none of the reduced models incorporate all aspects of the two-dimensional problem (as we indicated in the Introduction), and, consequently all begin to fail as the effects of the fluid mixing become more pronounced (i.e., in the limit $\mathcal{P} \rightarrow \infty$ ). This failure of the reduced models can surely come as no surprise. Neither can the indifference of the yield to the exact nature of the reduced model in the opposite limit, $\mathcal{P} \rightarrow 0$, where homogenisation becomes instantaneous, for reasons outlined above. The contribution of the present article is certainly not in demonstrating the success of any of the described models as $\mathcal{P} \rightarrow 0$ and none as $\mathcal{P} \rightarrow \infty$. Rather, its contribution is two-fold. First, we give for the first time accurate simulations of the two-stage reaction scheme (1) in a chaotic flow, across a range of Péclet numbers. Second, we give in quantitative terms an indication of the range in Péclet number over which one might hope that the reduced models correspond to the full two-dimensional simulations. It is not our thesis that those reduced models described here should ever be wholly adopted as a proxy for the full system. But reduced models similar to those evaluated here are used as a test-bed for theoretical advances, apparently on the tacit assumption that the insights gained will 'scale up'. The present results are offered as a quantitative 'reality check' on the extent to which such 
aspirations might hold good.

\section{Conclusions}

We have carried out accurate simulations of a two-stage competitive-consecutive chemical reaction in the liquid phase, chaotically mixed from an initially segregated initial state. We have computed the effect of fluid mixing on the yield for a range of Péclet numbers, and have compared these two-dimensional simulations with a variety of reduced models, to evaluate the predictive power of the latter, focusing on fast reactions. For such reactions, the well mixed model holds in the limit $\mathcal{P} \rightarrow 0$, as one might expect. For $\mathcal{P}>10$ the well mixed performs poorly, and for $\mathcal{P}>30$ so does a lamellar model that does not account for the fluid mixing. In order to model the effects of fluid mixing, a lamellar model requires some estimate of a typical stretching rate generated by the flow, and there can be wide variations in the predicted yield, depending on how this quantity is specified. The variation is greatest at large $\mathcal{P}$. The lamellar models give broadly the correct trend, that the yield diminishes as the effects of segregation and fluid mixing become more significant (i.e., as $\mathcal{P}$ increases), and give reasonable quantitative estimates of the yield for moderate $\mathcal{P}$, up to around 300. For larger $\mathcal{P}$, though, the lamellar models significantly overestimate the yield. However, the lamellar models that we have considered are relatively simple variants, and it remains to be seen whether more sophisticated lamellar models might improve over the versions presented here. These might involve modelling the effects of curvature of the lamellae, simulating a greater number of lamellae, with a nonuniform distribution of widths (reflecting the corresponding distribution of striation widths generated by the chaotic fluid flow).

\section{Appendix}

\section{A Early time behaviour at a single interface}

At $O\left(T^{0}\right)$ in $(25)-(26)$, we find that $\hat{A}_{0}^{\prime \prime}+2 \eta \hat{A}_{0}^{\prime}=0$ and $\hat{B}_{0}^{\prime \prime}+2 \eta \hat{B}_{0}^{\prime}=0$, for which the solutions satisfying the appropriate boundary conditions are 
$\hat{A}_{0}=\frac{1}{2} A_{0}(1-\operatorname{erf} \eta)$ and $\hat{B}_{0}=\frac{1}{2} B_{0}(1+\operatorname{erf} \eta)$. There are no products formed at this order.

At $O\left(T^{1}\right)$ in $(25)-(26)$, we find that

$$
\begin{aligned}
& \hat{A}_{1}^{\prime \prime}+2 \eta \hat{A}_{1}^{\prime}-4 \hat{A}_{1}=k_{1} \mathcal{P} A_{0} B_{0}\left(1-\operatorname{erf}^{2} \eta\right), \\
& \hat{B}_{1}^{\prime \prime}+2 \eta \hat{B}_{1}^{\prime}-4 \hat{B}_{1}=k_{1} \mathcal{P} A_{0} B_{0}\left(1-\operatorname{erf}^{2} \eta\right)
\end{aligned}
$$

subject to $\hat{A}_{1}, \hat{B}_{1} \rightarrow 0$ as $\eta \rightarrow \pm \infty$. Thus $\hat{A}_{1}=\hat{B}_{1}=-k_{1} \mathcal{P} A_{0} B_{0} \phi(\eta)$, where $\phi(\eta)$ is the (positive, even) function satisfying

$$
\phi^{\prime \prime}+2 \eta \phi^{\prime}-4 \phi=-\left(1-\operatorname{erf}^{2} \eta\right)
$$

subject to $\phi \rightarrow 0$ as $\eta \rightarrow \pm \infty$. In computing the leading-order effects of the mixing upon the reaction yield, we shall not need to compute $\phi(\eta)$ itself [15], although it will prove useful to note that integration of (A.3) over the interval $-\infty<\eta<\infty$ yields

$$
\int_{-\infty}^{\infty} \phi(\eta) \mathrm{d} \eta=\frac{1}{6} I, \quad \text { where } I=\int_{-\infty}^{\infty} 1-\operatorname{erf}^{2} \eta \mathrm{d} \eta \approx 1.5958
$$

From (21) and (22), it follows that

$$
\hat{R}_{1}=k_{1} \mathcal{P} A_{0} B_{0} \phi(\eta) \quad\left[=-\hat{A}_{1}=-\hat{B}_{1}\right]
$$

Although this expression gives the leading-order generation of $R$ at small times, it carries no information about the initial effects of the mixing upon the yield, so we turn to the next order in $T$ to determine this.

At $O\left(T^{2}\right)$, we find that $\hat{R}_{2}$ satisfies

$$
\hat{R}_{2}^{\prime \prime}+2 \eta \hat{R}_{2}^{\prime}-8 \hat{R}_{2}=4 \mathcal{P}\left(k_{1} \hat{A}_{0}+\left(k_{1}+k_{2}\right) \hat{B}_{0}\right) \hat{R}_{1}+8 k_{1} \mathcal{P}^{2} \mu \hat{A}_{0} \hat{B}_{0},
$$

where we have used the result that $\hat{R}_{1}=-\hat{A}_{1}=-\hat{B}_{1}$. Integration of this expression over the interval $-\infty<\eta<\infty$, gives

$$
\int_{-\infty}^{\infty} \hat{R}_{2}(\eta) \mathrm{d} \eta=-\frac{1}{30} k_{1} \mathcal{P}^{2} A_{0} B_{0}\left(k_{1} A_{0}+\left(k_{1}+k_{2}\right) B_{0}\right) I-\frac{1}{5} k_{1} \mathcal{P}^{2} \mu A_{0} B_{0} I .
$$


The first term on the right-hand side of this expression reflects reduced production of $R$ due to two factors: first, the depletion of $A$ and $B$; second, the loss of $R$ in the secondary reaction with $B$. The second term provides information about the effects of the fluid mixing. Thus the total amount of $R$ produced, per unit initial length in $y$, is

$$
\langle R\rangle \sim 2 k_{1} \mathcal{P} A_{0} B_{0} I T^{1 / 2}\left\{\frac{1}{6} T-\mathcal{P} T^{2}\left[\frac{1}{30}\left(k_{1} A_{0}+\left(k_{1}+k_{2}\right) B_{0}\right)+\frac{1}{5} \mu\right]\right\},
$$

where $\langle R\rangle \equiv \mathrm{e}^{\mu t} \int_{-\infty}^{\infty} R \mathrm{~d} x$. The exponential factor in the definition of $\langle R\rangle$ reflects the exponentially growing nature of the interface in the $y$-direction.

\section{References}

[1] O. Levenspiel, Chemical reaction engineering, second edition (John Wiley and Sons, New York, 1972).

[2] J. M. Ottino, Mixing and chemical reactions, Chem. Eng. Sci. 49 (1994) 4005.

[3] R. Chella and J. M. Ottino, Modelling of rapidly-mixed fast-crosslinking exothermic polymerizations, AIChE J. 29 (1983) 373.

[4] R. Chella and J. M. Ottino, Conversion and selectivity modifications due to mixing in unpremixed reactors, Chem. Eng. Sci. 39 (1984) 551.

[5] G. Metcalfe and J. M. Ottino, Autocatalytic processes in mixing flows, Phys. Rev. Lett. 72 (1994) 2875.

[6] F. J. Muzzio and M. Liu, Chemical reactions in chaotic flows, Chem. Eng. J. 64 (1996) 117.

[7] Z. Neufeld, C. López, E. Hernández-García and O. Piro, Excitable media in open and closed chaotic flows, Phys. Rev. E 66 (2002) 066208.

[8] Z. Neufeld, P. H. Haynes and T. Tél, Chaotic mixing induced transitions in reaction-diffusion systems, Chaos 12 (2002) 426.

[9] J. M. Zalc and F. J. Muzzio, Parallel-competitive reactions in a two-dimensional chaotic flow, Chem. Eng. Sci. 54 (1999) 1053.

[10] M. Giona, S. Cerbelli and A. Adrover, Quantitative analysis of mixing structures in chaotic flows generated by infinitely fast reactions in the presence of diffusion, J. Phys. Chem. 106 (2002) 5722. 
[11] J. R. A. Pearson, Diffusion of one substance into a semi-infinite medium containing another with second-order reaction, Appl. Sci. Res. 11 (1961) 321.

[12] W. E. Ranz, Applications of a stretch model to mixing, diffusion, and reaction in laminar and turbulent flows, AIChE J. 25 (1979) 41.

[13] M. J. Clifford, S. M. Cox and E. P. L. Roberts, Lamellar modelling of reaction, diffusion and mixing in a two-dimensional flow, Chem. Eng. J. 71 (1998) 49.

[14] M. J. Clifford, S. M. Cox and E. P. L. Roberts, Reaction and diffusion in a lamellar structure: the effect of the lamellar arrangement upon yield, Physica A 262 (1999) 294.

[15] S. M. Cox, M. J. Clifford and E. P. L. Roberts, A two-stage reaction with initially separated reactants, Physica A 256 (1998) 65.

[16] S. D. Fields and J. M. Ottino, Effect of striation thickness distribution on the course of an unpremixed polymerization, Chem. Eng. Sci. 42 (1987) 459.

[17] S. D. Fields and J. M. Ottino, Effect of stretching path on the course of polymerizations: applications to idealized unpremixed reactors, Chem. Eng. Sci. 42 (1987) 467.

[18] S. D. Fields and J. M. Ottino, Effect of segregation on the course of unpremixed polymerizations, AIChE J. 33 (1987) 959.

[19] F. J. Muzzio and J. M. Ottino, Evolution of a lamellar system with diffusion and reaction: a scaling approach, Phys. Rev. Lett. 63 (1989) 47.

[20] F. J. Muzzio and J. M. Ottino, Dynamics of a lamellar system with diffusion and reaction: scaling analysis and global kinetics, Phys. Rev. A 40 (1989) 7182.

[21] F. J. Muzzio and J. M. Ottino, Diffusion and reaction in a lamellar system: self-similarity with finite rates of reaction, Phys. Rev. A 42 (1990) 5873.

[22] D. M. Hobbs, M. M. Alvarez and F. J. Muzzio, Mixing in globally chaotic flows: a self-similar process, Fractals 5 (1997) 395.

[23] S. Cerbelli, J. M. Zalc and F. J. Muzzio, The evolution of material lines curvature in deterministic chaotic flows, Chem. Eng. Sci. 55 (2000) 363.

[24] M. Liu, R. L. Peskin, F. J. Muzzio and C. W. Leong, Structure of the stretching field in chaotic cavity flows, AIChE J. 40 (1994) 1273.

[25] J. G. Franjione and J. M. Ottino, Symmetry concepts for the geometric analysis of mixing flows, Phil. Trans. R. Soc. Lond. A 338 (1992) 301. 
[26] M. Liu, F. J. Muzzio and R. L. Peskin, Quantification of mixing in aperiodic chaotic flows, Chaos, Solitons and Fractals 4 (1994) 869.

[27] A. Adrover, S. Cerbelli and M. Giona, A spectral approach to reaction/diffusion kinetics in chaotic flows, Comp. Chem. Eng. 26 (2002) 125.

[28] M. Giona, S. Cerbelli and A. Adrover, Geometry of reaction interfaces in chaotic flows, Phys. Rev. Lett. 88 (2002) 024501.

[29] E. L. Paul and R. E. Treybal, Mixing and product distribution for a liquidphase, second-order, competitive-consecutive reaction, AIChE J. 17 (1971) 718.

[30] J. P. Boyd, Chebyshev and Fourier spectral methods, second edition (Dover, New York, 2001).

[31] J. Certaine, The solution of ordinary differential equations with large time constants, in Mathematical Methods for Digitial Computers, Eds A. Ralston and H. S. Wilf (1960) pp.128-132, Wiley, New York.

[32] S. M. Cox and P. C. Matthews, Exponential time differencing for stiff systems, J. Comp. Phys. 176 (2002) 430.

[33] D. Rothstein, E. Henry and J. P. Gollub, Persistent patterns in transient chaotic fluid mixing, Nature 401 (1999) 770.

[34] D. Beigie, A. Leonard and S. Wiggins, A global study of enhanced stretching and diffusion in chaotic tangles, Phys. Fluids A 3 (1991) 1039.

[35] Z. Koza and H. Taitelbaum, Motion of the reaction front in the $A+B \rightarrow C$ reaction-diffusion system, Phys. Rev. E 54 (1996) R1040.

[36] H. Taitelbaum, S. Havlin, J. E. Kiefer, B. Trus and G. H. Weiss, Some properties of the $A+B \rightarrow C$ reaction-diffusion system with initially separated components, J. Stat. Phys. 65 (1991) 873.

[37] H. Taitelbaum, Y.-E. L. Koo, S. Havlin, R. Kopelman and G. H. Weiss, Exotic behavior of the reaction front in the $A+B \rightarrow C$ reaction-diffusion system, Phys. Rev. A 46 (1992) 2151.

[38] H. Taitelbaum and Z. Koza, Anomalous kinetics of reaction-diffusion fronts, Philos. Mag. B 77 (1998) 1389.

[39] A. Yen, Z.-Y. Shi and R Kopelman, Early-time scaling for the reaction front of a ternary, $A+2 B \rightarrow C$, reaction-diffusion system, Phys. Rev. E 57 (1998) 2438 . 
[40] M. Lindberg and A. C. Rasmuson, Product concentration profile in strained reacting fluid films, Chem. Eng. Sci. 54 (1999) 483.

[41] I. M. Sokolov and A. Blumen, Mixing effects in the $A+B \rightarrow 0$ reaction-diffusion scheme, Phys Rev. Lett. 66 (1991) 1942.

[42] I. M. Sokolov and A. Blumen, Reactions in systems with mixing, J. Phys. A: Math. Gen. 24 (1991) 3687.

[43] M. M. Alvarez, F. J. Muzzio, S. Cerbelli, A. Adrover and M. Giona, Self-similar spatiotemporal structure of intermaterial boundaries in chaotic flows, Phys. Rev. Lett. 81 (1998) 3395.

[44] A. Adrover, M. Giona, F. J. Muzzio, S. Cerbelli and M. M. Alvarez, Analytic expression for the short-time rate of growth of the intermaterial contact perimeter in two-dimensional chaotic flows and Hamiltonian systems, Phys. Rev. E 58 (1998) 447.

[45] S. Cerbelli, M. M. Alvarez and F. J. Muzzio, Prediction and quantification of micromixing intensities in laminar flows, AIChE J. 48 (2002) 686.

[46] A. Schekochihin, S. Cowley, J. Maron and L. Malyshkin, Structure of small-scale magnetic fields in the kinetic dynamo theory, Phys. Rev. E 65 (2001) 016305.

[47] S. M. Cox and M. D. Finn, Behavior of the reaction front between initially segregated species in a two-stage reaction, Phys. Rev. E 63 (2001) 051102.

[48] Z. Koza, Asymptotic behaviour of initially separated $A+B$ (static) $\rightarrow 0$ reaction-diffusion systems, Physica A 240 (1997) 622.

[49] Z. Koza, Asymptotic properties of the $A+B \rightarrow 0$ reaction-diffusion front, Philos. Mag. B 77 (1998) 1437.

[50] H. A. Kusch, J. M. Ottino and D. M. Shannon, Analysis of impingement mixingreaction data: use of a lamellar model to generate fluid mixing information, Ind. Eng. Chem. Res. 28 (1989) 302. 


\section{Figure captions}

(1) Sine flow (8), (9). Left: flow during first half-period. Right: flow during second half-period.

(2) Poincaré maps for the sine flow with $T=0.8$ (left) and $T=1.6$ (right).

(3) Concentration field of $R$ after $\nu$ periods of the sine flow for $\nu=1,2, \ldots, 12$ (reading along rows, from top left to bottom right) at $T=0.8$ and $\mathcal{P}=$ $10^{4}$.

(4) Kinematic evolution of the interfaces $x=0$ and $x=1 / 2$ after, from left to right, one, two and three periods of the sine flow $(T=0.8)-\mathrm{cf}$. the first three plots of Figure 3.

(5) Concentration field of $R$ after $1,2, \ldots, 12$ periods of the sine flow (reading along rows, from top left to bottom right) at $T=1.6$ and $\mathcal{P}=10^{4}$. In each plot, white and black correspond, respectively, to maximum and minimum concentrations in that plot.

(6) Concentration field of $\mathrm{S}$ after $1,2, \ldots, 12$ periods of the sine flow (reading along rows, from top left to bottom right) at $T=1.6$ and $\mathcal{P}=10^{4}$. In each plot, white and black correspond, respectively, to maximum and minimum concentrations in that plot.

(7) As first two rows of Figure 5, except that all plots have common greyscale, with black corresponding to zero concentration and white to the concentration at large time. Note the loss of contrast with time.

(8) Plot of total amount $\langle R\rangle$ against $t$ for $\mathcal{P}=10^{4}$. Upper and lower curves correspond, respectively, to $T=1.6$ and $T=0.8$.

(9) Plot showing agreement between early-time prediction of $\langle R\rangle$ (dotted line $\mu=0$; dashed line $\mu=1.2$ ) and value computed from full two-dimensional simulations (solid line) for $T=1.6$ and $\mathcal{P}=1$.

(10) Application of the baker map to the lamellar array, carried out at discrete times $t=\nu \tau(\nu=1,2,3, \ldots)$. First the lamellae are squashed to half their width, then a mirror image set of lamellae is adjoined along $x=1 / 2$.

(11) Final yield of product $R$ as a function of Péclet number $\mathcal{P}$. Curves represent: full two-dimensional simulations (§3.2); well mixed model ( $\S 4.1)$; lamellar model with no stretch, $\mu=0$, and with stretch rate $\mu=1.2$ (§4.3.1); and baker map model with $\tau=\log 2 / 1.2$ (§4.3.2). 